\title{
Análise da associação entre saneamento e saúde nos estados brasileiros - estudo comparativo entre 2001 e 2006
}

\section{Analysis of the association between sanitation and health in Brazilian states - comparative study in from 2001 to 2006}

\author{
Júlio César Teixeira \\ Engenheiro Civil e de Segurança no Trabalho. Doutor em Saneamento, Meio Ambiente e Recursos Hídricos. Professor Adjunto do Departamento de \\ Engenharia Sanitária e Ambiental da Universidade Federal de Juiz de Fora (UFJF)
}

\section{Maria Helena Rodrigues Gomes}

Engenheira Civil. Doutora em Hidráulica e Saneamento pela Escola de Engenharia de São Carlos da Universidade de São Paulo (EESC/USP). Professora Adjunta do Departamento de Engenharia Sanitária e Ambiental da UFJF

Janaina Azevedo de Souza

Estudante de Engenharia em Engenharia Sanitária e Ambiental pela UFJF. Bolsista de Iniciação Científica da UFJF

\section{Resumo}

Em um contexto de baixo investimento em saneamento, Teixeira e Guilhermino (2006) estudaram a associação entre as condições de saneamento e os indicadores epidemiológicos nos estados brasileiros, utilizando dados secundários do banco de dados IDB (2003) do Ministério da Saúde. A partir de 2003, os investimentos em saneamento básico no Brasil aumentaram. Assim, o presente trabalho teve como objetivo testar a hipótese de que a ampliação da cobertura por serviços de saneamento básico no período entre 2001 e 2006 foi um investimento capaz de melhorar os indicadores de saúde nos estados brasileiros. Concluiu-se que houve melhoria nos indicadores de saúde, porém não-expressiva, sendo fundamental a utilização de indicadores epidemiológicos e de desenvolvimento social na decisão sobre a priorização de investimentos em saneamento no Brasil.

Palavras-chave: associação; saneamento; saúde; estados brasileiros; indicadores.

\section{Abstract}

In a context of poor investment in sanitation, Teixeira e Guilhermino (2006) studied the association between the sanitation conditions and the epidemiological indicators in the Brazilian states, based on secondary data from the database from IDB (2003) of the Ministry of Health. Since 2003, investments in basic sanitation in Brazil had an expressive increase. Thus, the present paper had as a purpose to test the hypothesis that the improvement in the coverage by basic sanitation services from 2001 to 2006 was an investment capable to improve the studied health indicators in the Brazilian states. It has been concluded that there was an improvement in the health indicators, which is still not expressive, being of the most importance the use of epidemiological and social development indicators in the priority of investments in basic sanitation in Brazil.

Keywords: association; sanitation; health; Brazilian states; indicators.

Endereço para correspondência: Júlio César Teixeira - Departamento de Engenharia Sanitária e Ambiental - Faculdade de Engenharia da Universidade Federal de Juiz de Fora - Sala 4.103 - Bairro São Pedro - 36036-900 - Juiz de Fora (MG), Brasil - E-mail: juliotei@terra.com.br - Tel.: (32) 3232-6342/ 3229-3419

Recebido: 16/12/10 - Aceito: 01/04/11 - Reg. ABES: 21609 


\section{Introdução}

O reconhecimento da importância do saneamento básico e de sua associação com a saúde humana remonta às mais antigas culturas. Ruínas de uma grande civilização ao Norte da Índia, com mais de 4.000 anos, indicam evidências da existência de hábitos higiênicos, incluindo a presença de banheiros e sistemas de coleta de esgotos sanitários nas edificações, além da drenagem nos arruamentos. Os egípcios dispunham de sistemas de drenagem de água e grandes aquedutos. Também destacam-se os cuidados com o destino dos dejetos na cultura creto-micênica e as noções de engenharia sanitária dos romanos (ROSEN, 1994).

Heller (1997) realizou vasta revisão da literatura disponível sobre a relação entre saneamento básico e saúde pública e consolidou o entendimento de que os estudos já realizados permitem afirmar que há melhora dos indicadores de saúde, com a melhoria da cobertura por serviços de saneamento básico - abastecimento de água potável, esgotamento sanitário, limpeza urbana e manejo de resíduos sólidos e drenagem das águas pluviais urbanas.

No Brasil, no início da década de 1990, a Lei 8.080 (Brasil, 1990), que dispõe sobre o funcionamento dos serviços de saúde no país, reconheceu em seu artigo $3^{\circ}$ que a saúde tem como fatores determinantes e condicionantes, entre outros, o saneamento básico. Logo, houve o reconhecimento legal da existência de uma relação de causalidade entre condições inadequadas de saneamento básico e o quadro epidemiológico existente.

Mais recentemente, a Lei 11.445 (BRASIL, 2007a), chamada Lei do Saneamento Básico, estabeleceu que os serviços públicos de saneamento básico serão prestados com base em 12 princípios fundamentais, destacando-se: a universalização do acesso; a integralidade dos diversos serviços de saneamento básico; a prestação dos serviços de abastecimento de água, esgotamento sanitário, limpeza urbana e manejo dos resíduos sólidos realizados de formas adequadas à saúde pública e à proteção do meio ambiente; e a disponibilidade, em todas as áreas urbanas, de serviços de drenagem e manejo das águas pluviais adequados à saúde pública e à segurança da vida e dos patrimônios público e privado.

Teixeira e Guilhermino (2006) realizaram um estudo da associação entre saneamento e saúde nos estados brasileiros, utilizando dados secundários do banco de dados "Indicadores e Dados Básicos para a Saúde 2003" (IDB, 2003) do Ministério da Saúde, e encontraram que a mortalidade infantil, a mortalidade proporcional por diarreia aguda em menores de cinco anos de idade e a mortalidade proporcional por doenças infecciosas e parasitárias para todas as idades estavam associadas com deficiências na cobertura por serviços de saneamento básico - água e esgotos - em 2001.

A partir de 2003, os investimentos em saneamento básico no Brasil aumentaram expressivamente com recursos do Orçamento Geral da União (OGU), do Fundo de Garantia por Tempo de
Serviço (FGTS) e do Programa de Aceleração do Crescimento (PAC). Neste contexto, o objetivo do presente trabalho foi desenvolver um estudo entre a associação entre indicadores epidemiológicos (taxa de mortalidade infantil, mortalidade proporcional por doença diarreica aguda em crianças menores de cinco anos de idade e mortalidade proporcional por doenças infecciosas e parasitárias para todas as idades) e cobertura por serviços de saneamento básico (água, esgotos e lixo) nos estados brasileiros, utilizando dados secundários do banco de dados "Indicadores e Dados Básicos para a Saúde 2008" (IDB, 2008) do Ministério da Saúde, visando testar a hipótese de que a ampliação da cobertura por serviços de saneamento básico nos estados brasileiros, no período entre 2001 e 2006, foi um investimento capaz de melhorar os indicadores de saúde da população brasileira.

\section{Metodologia}

\section{Delineamento epidemiológico}

O método epidemiológico empregado foi um estudo ecológico, que pode ser mais bem compreendido como o estudo epidemiológico que utiliza agregados populacionais como unidade de análise.

\section{Base de dados}

A base de dados foi composta por dados secundários provenientes da publicação "Indicadores e Dados Básicos para a Saúde 2008" (IDB, 2008), editada pela Rede Interagencial de Informações para a Saúde (RIPSA).

\section{Indicadores de saúde estudados}

- Taxa de mortalidade infantil: 2006 (número de óbitos infantis menores de um ano de idade por 1.000 nascidos vivos).

- Mortalidade proporcional por doença diarreica aguda em menores de cinco anos de idade: 2006 (\%).

- Mortalidade proporcional por doenças infecciosas e parasitárias para todas as idades: 2006 (\%).

\section{Fatores de risco estudados}

Cada um dos três indicadores de saúde estudado foi analisado por meio da correlação com vários outros indicadores, divididos em cinco classes de fatores de risco, a saber:

\section{Indicadores demográficos}

- Taxa de crescimento da população de 2001 a 2007 (\% de incremento populacional);

- Grau de urbanização, 2007 (\% da população urbana); 
- Taxa de fecundidade total, 2006 (número médio de nascidos vivos por mulher);

- Taxa padronizada de natalidade, 2006 (número de nascidos vivos por 1.000 habitantes);

- Esperança de vida ao nascer, 2007 (número de anos de vida esperados).

\section{Indicadores socioeconômicos}

- Taxa de analfabetismo, 2007 (\% na população de 15 anos ou mais de idade);

- Produto Interno Bruto (PIB) per capita, 2006 (R\$/habitante);

- Razão de renda, 2007 (número de vezes que a renda dos 20\% mais ricos supera a dos 20\% mais pobres em um estado);

- Proporção de pobres, 2007 (\% da população com renda familiar per capita de até meio salário mínimo);

- Taxa de desemprego, 2007 (\% da população economicamente ativa que se encontrava sem trabalho).

\section{Indicadores de morbidade e fatores de risco}

- Proporção de nascidos vivos de baixo peso ao nascer, 2006 (\% de nascidos com peso inferior a $2.500 \mathrm{~g}$ );

- Prevalência de déficit ponderal para a idade em crianças menores de cinco anos de idade, 2006 (\%);

- Prevalência de aleitamento materno exclusivo, 1999 (\%).

\section{Indicadores de recursos}

- Gasto público com saúde, como proporção do PIB, 2006 (\% do PIB);

- Gasto público com saneamento, como proporção do PIB, 2006 (\% do PIB).

\section{Indicadores de cobertura por serviços de saúde e de saneamento}

- Número de consultas médicas do Sistema Único de Saúde (SUS) por habitante, 2007 (consultas/habitante);

- Número de internações hospitalares no SUS por habitante, 2007 (internações/100 habitantes);

- Proporção de gestantes com acompanhamento pré-natal, 2006 (\% com seis e mais consultas);

- Cobertura vacinal no primeiro ano de vida, 2007 (\% de menores de um ano imunizados);

- Cobertura por sistemas de abastecimento de água, 2007 (\% da população urbana);

- Cobertura por sistemas de esgotamento sanitário, 2007 (\% da população urbana);

- Cobertura por serviços de coleta de lixo, 2007 (\% da população urbana).

Deve-se fazer a ressalva para o fato de que a metodologia utilizada para selecionar os fatores de risco no modelo não considerou se estas variáveis são independentes entre si. Assim, podem existir interações entre alguns fatores de risco como, por exemplo, a taxa de analfabetismo e o PIB per capita nos estados brasileiros, uma vez que a metodologia não excluiu possíveis interações.

\section{Análise de dados}

A análise epidemiológica foi desenvolvida segundo um processo evolutivo, em etapas, de tal forma a permitir a determinação progressiva das exposições efetivamente associadas aos indicadores de saúde estudados. Tal processo envolveu, em sequência, as seguintes atividades: análise descritiva dos indicadores para o conjunto das unidades da federação; análise de regressão linear simples entre os indicadores epidemiológicos versus indicadores demográficos, socioeconômicos, de morbidade e fatores de risco, de recursos, de cobertura por serviços de saúde e de saneamento, "um a um", de modo a avaliar as relações entre os indicadores epidemiológicos - variáveis dependentes e os demais indicadores - variáveis independentes - e pré-selecionar os indicadores a serem utilizados na regressão linear multivariada em nível de 10\% de significância ( $\mathrm{x}$ < 0,10); análise de regressão linear multivariada em que se procurou identificar as variáveis independentes estatisticamente associadas a cada indicador epidemiológico estudado em nível de 5\% de significância ( $\mathrm{p} \leq 0,05)$.

Foi utilizado o pacote estatístico SPSS 15.0 - Statistical Package for Social Sciences.

\section{Resultados}

\section{Taxa de mortalidade infantil}

No período entre os dois estudos, a taxa de mortalidade infantil no país caiu de 26,3 óbitos de crianças menores de um ano por 1.000 nascidos vivos, em 2001, para 20,7 óbitos de crianças menores de um ano por 1.000 nascidos vivos, em 2006, ou seja, uma redução de $21,3 \%$

Com os dados do IDB 2003, ao se processar a análise de regressão linear múltipla para a taxa de mortalidade infantil, os indicadores "esperança de vida ao nascer" e "cobertura por sistemas de esgotamento sanitário" apresentaram coeficiente $\beta$ negativo, mostrando uma relação inversamente proporcional com a taxa de mortalidade infantil nos estados brasileiros (TEIXEIRA; GUILHERMINO, 2006).

Ao se processar a análise de regressão linear múltipla para a taxa de mortalidade infantil nos estados brasileiros, referente aos dados do IDB 2008, encontrou-se um coeficiente $\mathrm{R}^{2}$ ajustado de 0,936, sendo que as variáveis que permaneceram no modelo final $(p \leq 0,05)$ foram: taxa de analfabetismo, razão de renda, prevalência de déficit ponderal para a idade em crianças menores de cinco anos de idade e cobertura por coleta de lixo com coeficiente $\beta$ positivo, mostrando relação diretamente proporcional com a taxa de mortalidade infantil nos estados. 
Em outra direção, os indicadores PIB per capita, gasto público com saúde como proporção do PIB e cobertura por sistemas de esgotamento sanitário apresentaram coeficiente $\beta$ negativo, mostrando uma relação inversamente proporcional com a taxa de mortalidade infantil (Tabela 1).

Surpreendeu o grande número de variáveis com valor p menor que $5 \%$ obtido quando a análise de regressão linear múltipla para a taxa de mortalidade infantil nos estados brasileiros foi realizada, como apresentado na Tabela 1. Em geral, apenas um número restrito de variáveis acaba tendo valor de $\mathrm{p}$ menor que 5\%. Uma hipótese para que sete variáveis tenham permanecido no modelo final estatisticamente associadas à taxa de mortalidade infantil é a existência de correlação entre algumas das variáveis com valor de p menor que 5\%.

Na Figura 1, observou-se que quanto maior a cobertura populacional por serviços adequados de esgotamento sanitário, menor é a mortalidade infantil em um estado da federação para os dados do IDB 2008. Já na Figura 2, observou-se que quanto maior o PIB per capita em um estado da federação, menor é a taxa de mortalidade infantil naquele estado. Ainda, na Figura 3, observou-se que quanto maior a taxa de analfabetismo, maior é a mortalidade infantil em um estado da federação para os dados do IDB 2008.

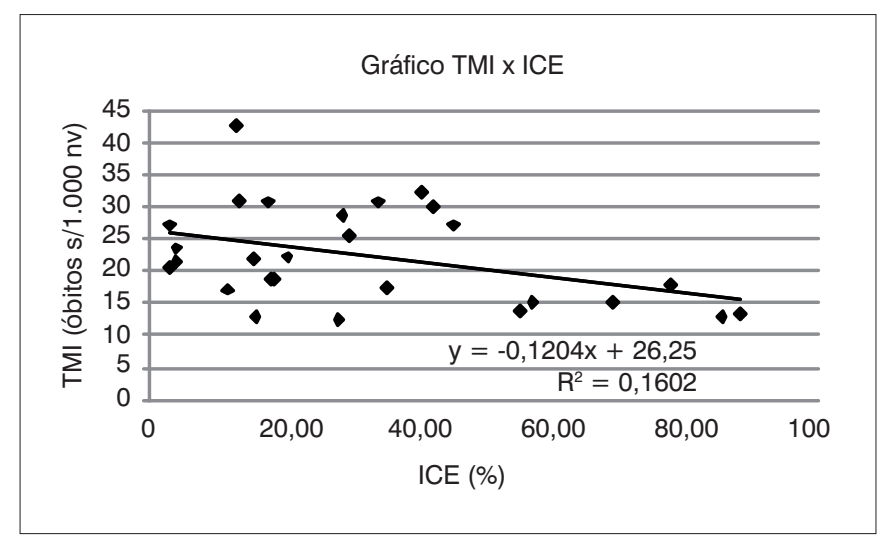

Figura 1 - Regressão linear simples entre a taxa de mortalidade infantil (TMI) e a cobertura por sistemas de esgotamento sanitário (ICE) nos estados brasileiros (IDB, 2008)

\section{Mortalidade proporcional por doença diarreica aguda em menores de cinco anos de idade}

Entre os dois estudos, 2001 e 2006, observou-se uma redução de $11,4 \%$ na mortalidade proporcional por doença diarreica aguda em menores de cinco anos de idade, a saber, redução de 4,4\%, em 2001, para 3,9\% do total de óbitos de crianças menores de cinco anos de idade, em 2006.

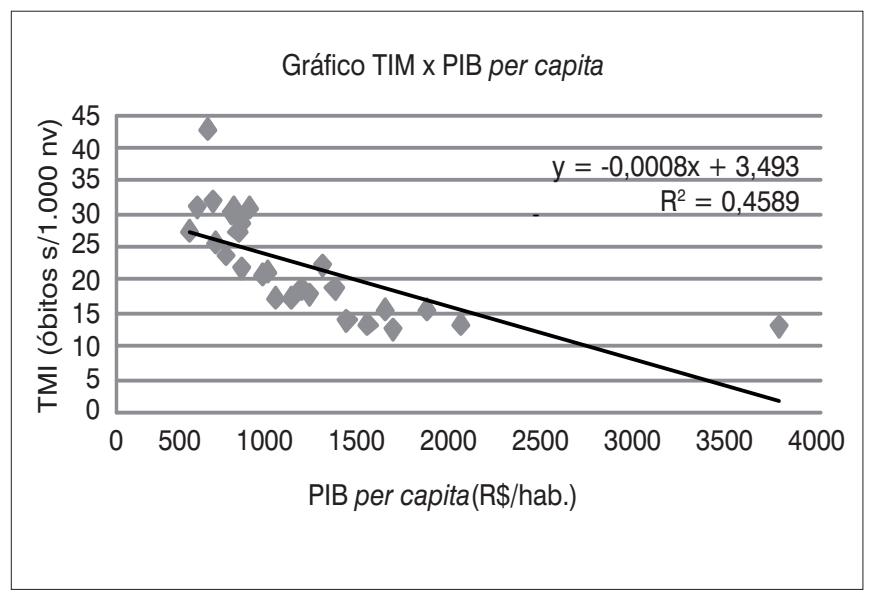

Figura 2 - Regressão linear simples entre a taxa de mortalidade infantil (TMI) e o Produto Interno Bruto (PIB) per capita nos estados brasileiros (IDB, 2008)

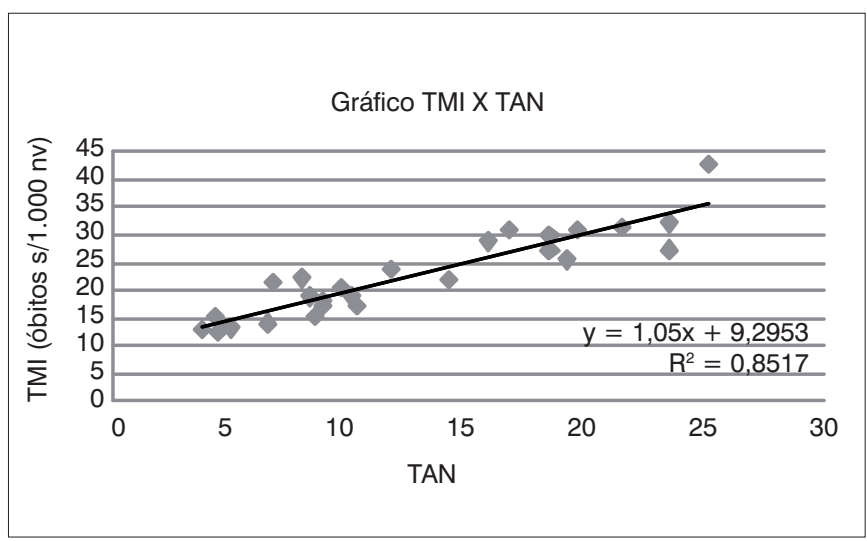

Figura 3 - Regressão linear simples entre a taxa de mortalidade infantil (TMI) e a taxa de analfabetismo nos estados brasileiros (IDB, 2008)

Tabela 1 - Regressão linear multivariada entre a taxa de mortalidade infantil e os indicadores demográficos, socioeconômicos, de morbidade e fatores de risco, de recursos e de cobertura por serviços de saúde e de saneamento nos estados brasileiros (IDB, 2008)

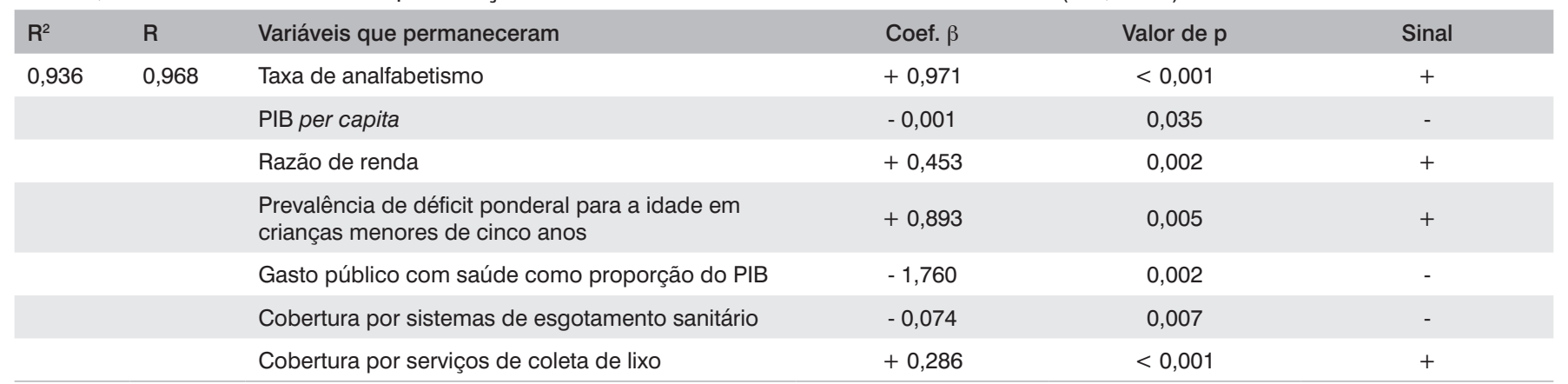

R: coeficiente de correlação de Pearson; $R^{2}$ : coeficiente de determinação. 
Segundo Teixeira e Guilhermino (2006), com os dados do IDB 2003 na regressão linear múltipla para a mortalidade proporcional por doença diarreica aguda em menores de cinco anos de idade, o indicador "cobertura por sistemas de esgotamento sanitário" apresentou coeficiente $\beta$ negativo, mostrando relação inversamente proporcional com a mortalidade proporcional por doença diarreica aguda em menores de cinco anos de idade. Já a proporção de pobres apresentou um coeficiente $\beta$ positivo, demonstrando uma relação diretamente proporcional com este tipo de mortalidade.

$\mathrm{Na}$ análise de regressão linear múltipla com os dados do IDB 2008 (Tabela 2), encontrou-se, como variáveis estatisticamente associadas à mortalidade proporcional por doença diarreica aguda em crianças menores de cinco anos, com $\mathrm{R}^{2}$ ajustado de 0,580, a proporção de pobres e gasto público com saneamento como proporção do PIB. A exemplo de 2003, a variável proporção de pobres apresentou um coeficiente $\beta$ positivo, demonstrando uma relação diretamente proporcional com a mortalidade por doença diarreica aguda em crianças menores de cinco anos. O gasto público com saneamento como proporção do PIB nos estados apresentou um coeficiente $\beta$ positivo também, ou seja, uma relação diretamente proporcional à mortalidade estudada.

A Figura 4 ilustra que quanto maior o gasto público com saneamento em um estado brasileiro em proporção ao PIB, maior a mortalidade proporcional por doença diarreica aguda em menores de cinco anos de idade para os dados do IDB 2008

Em uma primeira análise, o resultado apresentado na Figura 4 pode parecer equivocado. Pode, inclusive, levantar a hipótese da existência de um ponto outlier que estaria alterando o resultado, que é, em princípio, até certo ponto, contraditório. Assim, os autores processaram os dados eliminando o ponto outlier e o resultado se manteve. A partir deste procedimento metodológico, o resultado foi considerado correto e está relacionado com o critério adotado para priorização de investimentos em saneamento nos estados, como será discutido mais a frente.

\section{Mortalidade proporcional por doenças infecciosas e parasitárias para todas as idades}

Entre 2001 e 2006, houve um decréscimo de 5,45 para 4,92\% na taxa de mortalidade proporcional por doenças infecciosas e parasitárias para todas as idades, ou seja, uma redução percentual de 9,7\%. Com os dados do IDB 2003, na análise de regressão linear multivariada para mortalidade proporcional por doenças infecciosas e parasitárias, observou-se que a proporção de pobres apresentou coeficiente $\beta$ positivo, mostrando uma relação diretamente proporcional com a mortalidade proporcional por doenças infecciosas e parasitárias para todas as idades, enquanto a cobertura por sistemas de abastecimento de água apresentou um valor de $\beta$ negativo, mostrando uma relação inversamente proporcional com a mortalidade em estudo (TEIXEIRA; GUILHERMINO, 2006).

Para os dados do IDB 2008, quando da análise de regressão linear multivariada (Tabela 3), identificou-se um coeficiente $\mathrm{R}^{2}$ ajustado de 0,507 , sendo que as variáveis que permaneceram no modelo final foram a taxa de fecundidade total $(\mathrm{p}<0,001)$ e a cobertura por sistemas de abastecimento de água $(p=0,028)$. O indicador taxa de fecundidade total apresentou coeficiente $\beta$ positivo, mostrando uma relação diretamente proporcional com a mortalidade proporcional por doenças infecciosas e parasitárias para todas as idades. Já

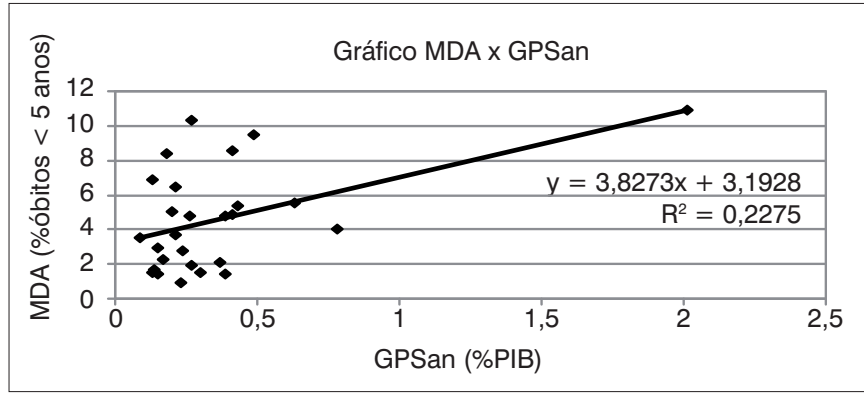

Figura 4 - Regressão linear simples entre a mortalidade proporcional por doença diarreica aguda em menores de cinco anos de idade (MDA) e gasto público com saneamento como proporção do PIB (GPSan) nos estados brasileiros (IDB, 2008)

Tabela 2 - Regressão linear multivariada entre a mortalidade proporcional por doença diarreica aguda em menores de cinco anos de idade e indicadores demográficos, socioeconômicos, de morbidade e fatores de risco, de recursos e de cobertura por serviços de saúde e de saneamento nos estados brasileiros (IDB, 2008)

$\begin{array}{lllccc}\mathbf{R}^{2} & \mathbf{R} & \text { Variáveis que permaneceram } & \text { Coef. } \beta & \text { Valor de } p & \text { Sinal } \\ 0,580 & 0,713 & \text { Proporção de pobres } & +0,124 & <0,001 \quad+ & + \\ & & \text { Gasto público com saneamento } & +2,596 & 0,021 & +\end{array}$

R: coeficiente de correlação de Pearson; $R^{2}$ : coeficiente de determinação.

Tabela 3 - Regressão linear multivariada entre a mortalidade proporcional por doenças infecciosas e parasitárias para todas as idades e indicadores demográficos, socioeconômicos, de morbidade e fatores de risco, de recursos e de cobertura por serviços de saúde e de saneamento nos estados brasileiros (IDB, 2008)

$\begin{array}{lllll}\mathbf{R}^{2} & \mathbf{R} & \text { Variáveis que permaneceram } & \text { Coef. } \beta & \text { Sinal } \\ 0,507 & 0,712 & \text { Taxa de fecundidade total } & +1,310 \\ & & \text { Cobertura por sistemas de abastecimento de água } & -0,342 & 0,001\end{array}$

R: coeficiente de correlação de Pearson; $R^{2}$ : coeficiente de determinação. 


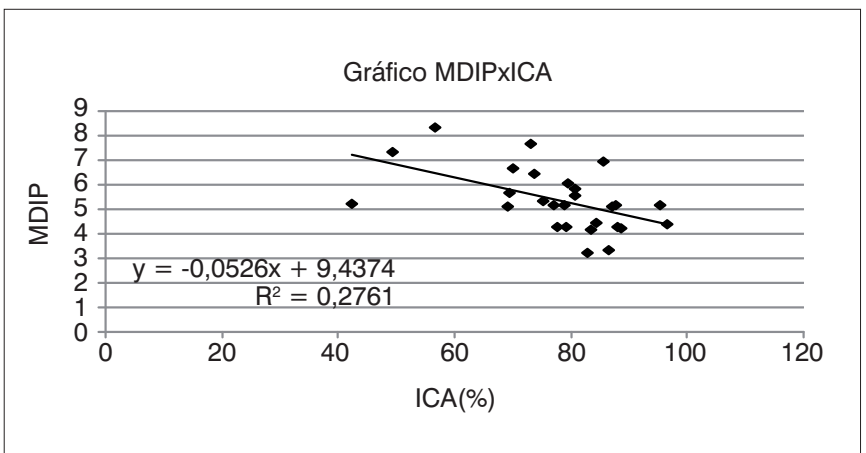

Figura 5 - Regressão linear simples entre a mortalidade proporcional por doenças infecciosas e parasitárias para todas as idades (MDIP) e a cobertura por redes de abastecimento de água (ICA) nos estados brasileiros (IDB, 2008)

o indicador cobertura por sistemas de abastecimento de água apresentou um valor de $\beta$ negativo, mostrando uma relação inversamente proporcional com o indicador em estudo.

Na Figura 5, observa-se que quanto maior a cobertura populacional por serviços adequados de abastecimento de água, menor é a mortalidade proporcional por doenças infecciosas e parasitárias para todas as idades nos estados da federação.

\section{Discussão}

"A mortalidade infantil, apesar de não considerar uma parte significativa de crianças passíveis de serem afetadas pelas questões do saneamento - as maiores de um ano -, mostra-se como um importante indicador epidemiológico para analisar o impacto das intervenções do saneamento na saúde humana. Trata-se de um indicador universal, padronizado há muitos anos e utilizado em todo o mundo para aferir o nível de desenvolvimento da sociedade" (COSTA et al., 2002).

Neste estudo, observou-se que a taxa de analfabetismo nos estados apresentou correlação positiva com a taxa de mortalidade infantil nas unidades da federação na análise multivariada. Este achado está de acordo com o descrito por Costa et al. (2003), no qual a taxa de analfabetismo apresentou forte correlação positiva com o coeficiente de mortalidade infantil no período de 1990 a 1998 no Brasil.

O PIB per capita apresentou uma correlação inversamente proporcional com a mortalidade infantil na análise multivariada, indicando que os estados com menor renda per capita no país apresentam maiores taxas de mortalidade infantil, corroborado pelo estudo de Victora (2001).

Ainda, no presente estudo, observou-se correlação positiva entre razão de renda e taxa de mortalidade infantil. Como já havia sido registrado por Godinho e Rodrigues (2001), há uma tendência de acréscimo da taxa de mortalidade infantil nas unidades da federação em que a concentração de renda é mais acentuada.
Notou-se também uma correlação positiva entre o déficit ponderal para a idade em crianças menores de cinco anos com a mortalidade infantil no país. Este resultado é consistente com as informações disponíveis para o Nordeste, onde são registradas as taxas mais elevadas de mortalidade infantil, da desnutrição energético proteica e da inadequação dos indicadores sociais (ASSIS et al., 2007).

Identificou-se, ainda, que o gasto público com saúde como proporção do PIB apresentou correlação negativa com a taxa de mortalidade infantil, provavelmente devido ao fato de que o aumento dos gastos em saúde nos estados após a implantação do Pacto Nacional pela Redução da Mortalidade Materna, Neonatal e Infantil (BRASIL, 2004) contribuiu para o desenvolvimento de estratégias de atenção voltadas para a redução da mortalidade infantil, resultando no aumento de coberturas vacinais, de assistência pré-natal e de consultas médicas.

O indicador "cobertura por sistemas de esgotamento sanitário" apresentou um coeficiente $\beta$ negativo, mostrando uma correlação inversamente proporcional com a taxa de mortalidade infantil para o estudo com os dados do IDB 2008. No estudo com dados do IDB 2003 (TEIXEIRA; GUILHERMINO, 2006), a cobertura por sistemas de esgotamento sanitário também apresentou relação inversamente proporcional com a taxa de mortalidade infantil. Esses dois resultados sugerem que os investimentos realizados na ampliação em sistemas de esgotamento sanitário, no período entre 2001 e 2006, não foram suficientes para eliminar a influência da baixa cobertura por sistemas de esgotamento sanitário sobre a mortalidade infantil no país.

A cobertura por serviços de coleta de lixo, na regressão linear simples, apresentou correlação negativa com a mortalidade infantil. Porém, na análise de regressão multivariada, teve o seu sinal invertido, ou seja, apresentou correlação positiva. Deve-se ressaltar que a unidade de análise é a unidade federativa e não indivíduos. Neste tipo de estudo - delineamento ecológico -, pode acontecer que o aumento da cobertura por coleta de lixo acarrete o aumento da mortalidade infantil, pois os dados são agregados. O fato de os dados serem agregados pode levar a resultados falsos, como este, o que é chamado de falácia ecológica.

Quanto à mortalidade proporcional por doença diarreica aguda em menores de cinco anos de idade, nos dois estudos, IDB 2003 e IDB 2008, encontrou-se uma correlação diretamente proporcional com a proporção de pobres nos estados da federação, ou seja, percentual da população residente em uma unidade da federação com renda familiar mensal per capita de até meio salário mínimo. Portanto, mais uma vez, reforça-se o entendimento que a mortalidade por doença diarreica aguda em menores de cinco anos de idade é uma doença típica da pobreza como já registrado por vários autores, tais como Teixeira e Heller (2005).

Encontrou-se, neste estudo, uma correlação diretamente proporcional entre a mortalidade proporcional por doença diarreica aguda 
em menores de cinco anos de idade e o gasto público com saneamento nos estados como proporção do PIB. O resultado foi coerente com o critério de alocação de recursos do FGTS para financiar obras de saneamento, adotado em 2006, que priorizaram investimentos em municípios e estados de acordo com a posição de cada projeto de investimento em um ranking nacional elaborado pelo Ministério das Cidades. Este ranking se baseou em um sistema de pontuação que considerou critérios como a taxa de mortalidade infantil do local, a mortalidade por diarreia aguda em menores de cinco anos de idade, a cobertura populacional por redes de água e de esgoto, a incidência de dengue e a substituição de lixões por aterros sanitários. As obras com projetos de engenharia prontos e com licença ambiental aprovada também ganhavam mais pontos no modelo de priorização adotado em 2006 (BRASIL, 2007b).

Quanto às doenças infecciosas e parasitárias, nos dois estudos, encontrou-se uma correlação inversamente proporcional entre a mortalidade proporcional por doenças infecciosas e parasitárias para todas as idades e a cobertura por redes de abastecimento de água. Tal resultado é corroborado por Libânio, Chernicharo e Nascimento (2005), os quais identificaram que os estados brasileiros com melhores indicadores de saúde - menores taxas de mortalidade e morbidade por doenças associadas à poluição hídrica - situam-se entre aqueles que apresentam os maiores índices de cobertura por rede de abastecimento de água, ou seja, abastecimento de água superior a $60 \%$ da população residente no estado.

Em relação à correlação diretamente proporcional entre a taxa de fecundidade total - número médio de nascidos vivos por mulher - e a mortalidade proporcional por doenças infecciosas e parasitárias para as idades observada neste estudo, tal correlação se explica pelo fato de que os estados com maiores taxas de fecundidade são os estados com condições mais precárias de saneamento, situados nas regiões Norte e Nordeste do país, onde as doenças infecciosas e parasitárias se constituem em causas importantes de mortes na infância.

\section{Conclusão}

Observou-se que existem diversos indicadores associados com a queda da taxa de mortalidade infantil nos estados brasileiros como, por exemplo, taxa de analfabetismo, desigualdade social caracterizada pela razão de renda, desnutrição crônica em crianças menores de cinco anos e gasto público em saúde. No entanto, uma única variável apareceu em ambos os estudos: cobertura por sistemas de esgotamento sanitário. Este resultado sugere que a baixa cobertura por esgotamento sanitário nos estados brasileiros, apenas 45,7\% das residências brasileiras eram atendidas por redes de esgoto em 2008 (IBGE, 2010), continua contribuindo para a mortalidade infantil no país.

A análise conjunta dos resultados de diferentes estudos nos diversos estados do território nacional demonstrou que a mortalidade proporcional por doença diarreica aguda, em menores de cinco anos de idade, continua sendo uma doença associada aos estados mais pobres da federação que, por sua vez, em 2006, foram os estados que mais receberam investimentos em obras de saneamento básico no âmbito do PAC (BRASIL, 2007b).

Observou-se, ainda, com os resultados dos estudos com o IDB 2003 e com o IDB 2008, que quanto maior a mortalidade proporcional por doenças infecciosas e parasitárias para todas as idades nos estados brasileiros, menor a cobertura por sistemas de abastecimento de água. Esta constatação sugere que a mortalidade por doenças infecciosas e parasitárias é resultante de inúmeras enfermidades relacionadas à contaminação da água para consumo humano por microorganismos patogênicos à saúde, que podem ser reduzidas com a universalização da cobertura populacional com sistemas de abastecimento de água de qualidade confiável.

Por fim, concluiu-se que, no período de 2001 a 2006, houve melhoria nos indicadores de saúde estudados, porém não foi expressiva, sendo fundamental a perene utilização de indicadores epidemiológicos e de desenvolvimento social na decisão sobre a priorização de investimentos em saneamento básico no país.

\section{Referências}

ASSIS, A.M.O. et al. Desigualdade, pobreza e condições de saúde e nutrição na infância no Nordeste brasileiro. Cadernos de Saúde Pública, v. 23, n. 10, p. 2337-2350, 2007.

BRASIL. IDB 2008. Indicadores e Dados Básicos para a Saúde 2008. Disponível em: <http://tabnet.datasus.gov.br/cgi/idb2008/matriz.htm>. Acesso em 01 jul. 2010

. Lein $n^{\circ} 11.445$, de 5 de janeiro de 2007. 2007a. Estabelece diretrizes nacionais para o saneamento básico; altera as Leis nos 6.766 , de 19 de dezembro de 1979, 8.036, de 11 de maio de 1990, 8.666, de 21 de junho de 1993, 8.987, de 13 de fevereiro de 1995; revoga a Lei $n^{\circ} 6.528$, de 11 de maio de 1978; e dá outras providências. Publicado no Diário Oficial da União - DOU de 8.1.2007 e retificado no DOU de 11.1.2007.

Programa de Aceleração do Crescimento - PAC. $2^{\circ}$ Balanço 2007. 2007b. Disponível em: <http://www.brasil.gov.br/pac/relatorios/ nacionais/2o-balanco >. Acesso em 25 nov. 2010.

Ministério da Saúde. Pacto Nacional pela Redução da Mortalidade Materna, Neonatal e Infantil. Brasília: Secretaria de Atenção à Saúde, 2004.

IDB. 2003. Indicadores e Dados Básicos para a Saúde 2003. 
Disponível em: < http://tabnet.datasus.gov.br/cgi/idb2003/matriz.htm>. Acesso em 01 jul. 2010

Lei $n^{\circ}$ 8.080, de 19 de setembro de 1990. Dispõe sobre as condições para a promoção, proteção e recuperação da saúde, a organização e o funcionamento dos serviços correspondentes e dá outras providências. Publicado no Diário Oficial da União - DOU de 20.9.1990.

COSTA, M.C.N. et al. Mortalidade infantil no Brasil em períodos recentes de crise econômica. Revista de Saúde Pública, v. 37, n. 6, p. 699-706, 2003.

COSTA, S.S. et al. Indicadores sanitários como sentinelas na prevenção e controle da mortalidade infantil - uma experiência utilizando o SISAGUA. In: XXVIII Congreso Interamericano de Ingeniería Sanitaria y Ambiental, Anais... Cancun: AIDIS, 2002.

GODINHO, R.E.; RODRIGUES, E.M. Caracterização das unidades da Federação e nível de mortalidade infantil. 2001. Disponível em: <http:// www.abep.nepo.unicamp.br/iussp2001/cd/GT_Pop_Saude_Godinho_ Rodrigues_Text.pdf >. Acesso em 16 nov. 2010.

Heller, L. Saneamento e saúde. Brasília: OPAS/OMS, 1997. 97p.
Instituto Brasileiro de Geografia e Estatística - IBGE. Pesquisa Nacional de Saneamento Básico de 2008. Rio de Janeiro: IBGE, 2010.

LIBÂNIO, P.A.C.; CHERNICHARO, C.A.L.; NASCIMENTO, N.O. A dimensão da qualidade da água: avaliação da relação entre indicadores sociais, de disponibilidade hídrica, de saneamento e de saúde pública. Revista de Engenharia Sanitária e Ambiental, v. 10, n. 3, p. 210-228, 2005.

ROSEN, G. Uma história da Saúde Pública. $2^{\mathrm{a}}$ ed. São Paulo: Editora UNESP, 1994. 423p.

TEIXEIRA, J.C.; GUILHERMINO, R.L. Análise da associação entre saneamento e saúde nos estados brasileiros, empregando dados secundários do banco de dados Indicadores e Dados Básicos para a Saúde - IDB 2003. Revista de Engenharia Sanitária e Ambiental, v. 11, n. 3, p. 277-282, 2006

TEIXEIRA, J.C; HELLER, L. Fatores ambientais associados à diarréia infantil em áreas de assentamento subnormal em Juiz de Fora, Minas Gerais. Revista Brasileira de Saúde Materno-infantil, v. 5, n. 4, p. 449-455, 2005.

VICTORA, C.G. (2001) Intervenções para reduzir a mortalidade infantil pré-escolar e materna no Brasil. Revista Brasileira de Epidemiologia, v. 4 , n. 1, p. 3-69, 2001 